\title{
Estudo experimental de linfonodo sentinela na mama da cadela com azul patente e Tecnécio Tc99m ${ }^{1}$
}

\author{
Luiz Gonzaga Porto Pinheiro ${ }^{2}$ \\ Manoel Odorico de Moraes ${ }^{3}$ \\ Aléssia Holanda Soares ${ }^{4}$ \\ Adriano Jorge Teixeira Lopes ${ }^{5}$ \\ Maria Amélia S. Pessoa Naguére ${ }^{6}$ \\ Francisco Átila de Lira Gondim ${ }^{7}$ \\ Carla Barbosa Brandão ${ }^{8}$ \\ Daiana Ciléa Honorato Nascimento ${ }^{8}$ \\ João Paulo Holanda Soares ${ }^{8}$ \\ João Marcos de Meneses e Silva ${ }^{8}$
}

\begin{abstract}
Pinheiro LGP, Moraes MO, Soares AH, Lopes AJT, Naguére MASP, Gondim FAL, Brandão CB, Nascimento DCH, Soares JPH, Silva JMM. Estudo experimental de linfonodo sentinela na mama da cadela com azul patente e Tecnécio Tc99m Acta Cir Bras [serial online] 2003 Nov-Dez;18(6). Disponível em URL: http://www.scielo.br/acb.
\end{abstract}

RESUMO - Pesquisa do Linfonodo Sentinela (LS) tem se mostrado efetiva na avaliação axilar nas portadoras de neoplasia mamária. O LS negativo torna desnecessário o esvaziamento axilar. Objetivo: Identificar o LS da região subareolar da mama em cadelas utilizando corante azul patente (AP), Tecnécio Tc99m ou a associação de ambas as técnicas e compará-las quanto à sensibilidade na detecção do linfonodo sentinela. Métodos: Foram estudadas dezessete cães fêmeas. Um total de 55 mamas foram analisadas. Utilizou-se AP $(2,5 \% / 0,5 \mathrm{ml}) \mathrm{e} / \mathrm{ou} \mathrm{Tc} 99 \mathrm{~m}(1,0 \mathrm{mC} /$ $0,8 \mathrm{ml}$ ) para identificação do LS. A aplicação do Tc99m era realizada duas horas antes da realização do experimento. O AP era injetado na região subareolar da mama. Localizado o LS, realizava-se sua exérese. Resultados: Dos 44 linfonodos em que se utilizou AP, pôde-se verificar que 40 estavam corados (90,90\%). Dos 48 linfonodos em que se utilizou Tc99m, 47 linfonodos estavam radioativos $(97,91 \%)(\mathrm{p}=0.18 ; \mathrm{k}=-0.067)$. Nas 37 mamas em que se associou $\mathrm{AP}$ ao Tc99m, apesar de 02 linfonodos não estarem corados, todos estavam radioativos (AP vs AP+Tc: $\mathrm{p}=0.12$; $\mathrm{k}=0.083$; Tc vs AP+Tc: $\mathrm{p}=1.0 ; \mathrm{k}=0.018$ ). Conclusão: $\mathrm{O}$ Tc99m e o corante azul patente, isolados ou associados, prestam-se à identificação do LS da mama do animal.

DESCRITORES - Biópsia de linfonodo sentinela. Câncer da mama. Cirurgia. Modelos animais.

\section{Introdução}

O estudo da drenagem linfática no planejamento terapêutico do câncer de mama começou com Henry François Le Dran, que nos meados do Séc. XVIII descreveu a progressão da doença por via linfática. Aplicando estes conhecimentos Halsted, no Séc. XIX, desenvolveu a mastectomia com ressecção dos músculos peitorais e o esvaziamento dos linfonodos axilares, denominada ressecção em monobloco. Foi o primeiro tratamento que permitiu a curabilidade desta neoplasia ${ }^{1}$. Esses princípios aplicam-se a outros tumores malignos, tais como melanomas, neoplasias uterinas, gástricas, intestinais, repetindo-se os resultados de curabilidade num percentual significativo de pacientes.
Os estudos das cadeias linfáticas por Sappey e, posteriormente, por Haagensen $^{2}$ dão o suporte anatômico para o entendimento do processo de disseminação linfática dos cânceres. A estratégia do tratamento cirúrgico dos tumores baseados nesses princípios perdurou por aproximadamente um século. Veronesi e col. ${ }^{5}$ mostraram a viabilidade de cura mediante ressecções mamárias parciais,

1. Trabalho realizado na Universidade Federal do Ceará (UFC) - Faculdade de Medicina - Departamento de Cirurgia - Laboratório de Cirurgia Experimental.

2. Prof. Dr. Adjunto do Depto. de Cirurgia da Faculdade de Medicina da UFC.

3. Prof. Dr. Adjunto do Depto. de Farmacologia da Faculdade de Medicina da UFC.

4. Cirurgiã Geral, aluna do curso de Especialização em Mastologia da Faculdade de Medicina da UFC.

5. Especialista em Medicina Nuclear, Diretor do Instituto de Medicina Nuclear - Fortaleza-CE.

6. Especialista em Medicina Nuclear.

7. Prof. Adjunto do Curso de Medicina Veterinária da UECE.

8. Acadêmicos de Medicina da UFC. 
associadas a esvaziamento axilar, radioterapia e quimioterapia (QUART). Além disso, chamaram a atenção, também, para o alto número de esvaziamentos axilares desnecessários em pacientes portadores de câncer de mama, quando clinicamente não se palpavam linfonodos. Mostrou ainda que havia um princípio de progressão escalonada da colonização dos linfonodos axilares nas neoplasias mamárias (níveis um, dois e três) e opcionais para cadeia da mamária interna. Cabanas ${ }^{3}$, no mesmo período, chamava atenção para a progressão de câncer de pênis que, a partir do órgão de origem, comprometia os linfonodos inguinais e, posteriormente, o linfonodo de Coclet, embaixo da arcada inguinal. A utilização de métodos anátomo-funcionais, utilizando azul patente injetado no sítio tumoral, demonstrou que havia de fato a possibilidade de identificar uma via preferencial linfática para drenagem de células melanomatosas para um linfonodo específico (sentinela). Giuliano e cols ${ }^{4}$, utilizando o mesmo sistema, associaram também substância radioativa (Tecnécio), que, aplicado na região tumoral, demonstrou a possibilidade de se identificar o sítio de drenagem inicial das neoplasias mamárias (linfonodo sentinela), permitindo o estudo prévio deste para a indicação ou não do esvaziamento axilar. A identificação do sítio preferencial de drenagem, que, quando não colonizado, tem se associado à ausência de neoplasia em todos os linfonodos do restante da cadeia $^{4,5}$, reforça a posição de que é desnecessária a retirada de toda a cadeia nesta circunstância. Em relação ao câncer de mama a técnica é aplicada nos casos iniciais (estadios clínicos T1 e T2).

Os vasos linfáticos provenientes das mamas anteriores da cadela, sobretudo dos três primeiros pares, drenam para os linfonodos axilares propriamente ditos. Estes estão localizados em uma massa de gordura na face medial da porção distal do músculo redondo maior na região da primeira e segunda costela. Eles estão situados ventralmente à artéria e veia toracodorsal. O tamanho dos linfonodos varia de 0,5 a $5,0 \mathrm{~cm}$. Os vasos linfáticos provenientes das mamas posteriores da cadela, sobretudo dos últimos três pares, drenam para os linfonodos inguinais superficiais (linfonodos mamários). Estes estão localizados aproximadamente 3 a $4 \mathrm{~cm}$ cranialmente ao osso púbico na face dorsolateral das glândulas mamárias. Geralmente, há dois linfonodos presentes, embora algumas vezes três ou quatro possam ser localizados, variando de 0,5 a 2,0 cm de comprimento 6 . O presente trabalho tem o objetivo de identificar o linfonodo sentinela da região retroareolar da mama em cadelas utilizando corante azul patente, Tecnécio ou a associação de ambas as técnicas e compará-las quanto à sensibilidade na detecção do linfonodo sentinela.

\section{Métodos}

Foram estudadas dezessete cães fêmeas, pesando aproximadamente quinze quilos, sem raça definida, cedidas pelo Centro de Controle de Zoonoses da Prefeitura de Fortaleza (Fortaleza-Ceará-Brasil), no período entre janeiro e abril de 2003. Estes animais faziam parte de um grupo que estavam com sacrifício programado.

Os animais foram anestesiados por via intramuscular, com Ketamin-Sâ (Cloridato de Cetamina $50 \mathrm{mg} / \mathrm{mL}$, em solução injetável de $10 \mathrm{~mL}$, sendo $10 \mathrm{mg} / \mathrm{Kg} \mathrm{IM}$ ), imediatamente antes do experimento e recebiam dose complementar de acordo com o plano anestésico.

Um total de 68 mamas foi estudado, preferencialmente o par anterior e/ou posterior. Deste total, 13 mamas foram excluídas da análise por problemas técnicos, perfazendo um total de 55 mamas analisadas. As mamas foram estudadas mediante aplicação de Corante Azul Patente V (Guerbert 2,5\% - 0,5 ml) e de tecnécio (Tc99m, substância radioativa a ser detectada pelo medidor "Gyger"), diluído em $0,8 \mathrm{~mL}$ de solução de fitato, com total de 0,8 milicurrie em cada área em estudo. A aplicação do tecnécio Tc99m era realizada em quatro pontos na região areolar das mamas anteriores e posteriores (dois pares de mamas) duas horas antes da realização do experimento. O corante azul patente era injetado na região subpapilar, $0,5 \mathrm{ml} \mathrm{em}$ ponto único, de cada mama. Aguardavase cerca de 15 minutos para iniciar-se a pesquisa do linfonodo sentinela.

Utilizava-se o aparelho "GPS Navigator" (Probe) para a detecção da radiação e conseqüente identificação do local de injeção do fármaco e do provável local de drenagem, ou seja, linfonodo sentinela de cada mama estudada. Realizava-se incisão cirúrgica no ponto considerado "quente".
A partir daí, realizava-se inspeção visual à procura de vias de drenagem coradas de azul, bem como se utilizava a sonda à medida que a diérese avançava. Localizado o linfonodo de drenagem, realizava-se sua exérese, medindo-se sua radioatividade ex-vivo, assim como se visualizando a presença de coloração azul. Procedia-se, então, a hemostasia e fechamento da incisão por planos.

Após o término do experimento, os animais sedados eram encaminhados ao Centro de Zoonoses para o sacrifício que era realizado utilizando-se Cloridato de Cetamina (Ketamin-Sâ) na dose de $10 \mathrm{mg} /$ $\mathrm{Kg}$ de peso e injeção intracardíaca de 1 mL de Cloreto de Potássio a $19,1 \%$. Os dados foram analisados utilizando-se o Teste Exato de Fischer e o coeficiente de concordância (Kappa). Estabeleceu-se em $5 \%$ o nível de significância $(\mathrm{p}<0,05)$.

\section{Resultados}

Utilizou-se corante azul patente associado a Tecnécio 99m em 37 mamas, tendo sido identificados 35 linfonodos corados e 2 não corados, sendo que todos os linfonodos foram identificados pelo gama-probe (Figura 1).

Em 11 mamas utilizou-se apenas Tecnécio Tc $99 \mathrm{~m}$, sendo que foram identificados 10 linfonodos pelo gama-probe, bem como um linfonodo apresentava índice radioativo muito baixo (identificação anatômica). Em outras 7 mamas utilizou-se apenas corante azul patente, tendo sido identificados 5 linfonodos corados e 2 linfonodos não corados (identificação anatômica) (Figuras 2 e 3).

Deste modo, dos 44 linfonodos em que se utilizou corante azul patente (AP), pôde-se verificar que 40 estavam corados (90,90\%). Dos 48 linfonodos em que se utilizou Tecnécio Tc99m, 47 linfonodos estavam com radioatividade detectável $(97,91 \%)(\mathrm{p}=0,18 ; \mathrm{k}=-0,067)$. Nas 37 mamas em que se associou corante azul patente a Tecnécio Tc $99 \mathrm{~m}$, apesar de 2 linfonodos não estarem corados, pôde-se identificálos em $100 \%$ dos casos, tendo em vista a acentuada positividade do Tecnécio Tc99m (AP vs AP+Tc: $\mathrm{p}=0,12 ; \mathrm{k}=0,083 ; \mathrm{Tc}$ vs $A P+T c: p=1,0 ; k=0,018)$. Não houve diferença significante entre os grupos em relação à identificação do linfonodo sentinela na mama. 


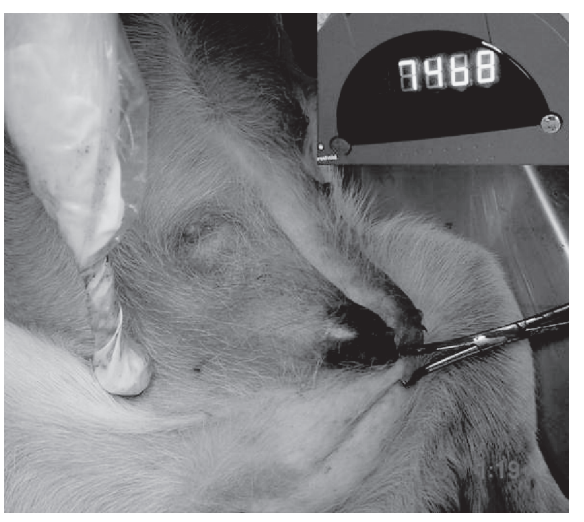

FIGURA 1 - Identificação do linfonodo sentinela pelo "Gama-Probe".

\section{Discussão}

A técnica do Linfonodo Sentinela (LS) tem se mostrado efetiva na avaliação do status nodal de pacientes com câncer de mama inicial (estágios clínicos T1 e T2) e melanoma maligno, por limitar a extensão da operação para estadiamento tumoral ${ }^{7-9}$.

O esvaziamento radical dos linfonodos axilares na terapêutica do câncer de mama está relacionado a uma série de complicações que influem sobremaneira na qualidade de vida da paciente: seroma, linfedema, distúrbios locais de sensibilidade, maior permanência hospitalar, dor crônica, dificuldade de movimentação do braço ${ }^{10,11}$, tendo sido inclusive reportados casos de indução de tumores malignos (angiossarcoma) $)^{12}$.

A técnica "ótima" para a pesquisa do linfonodo sentinela ainda está em estu$\mathrm{do}^{13}$. Alguns trabalhos da literatura demonstram serem os dois métodos adequados para a identificação do linfonodo sentinela. $\mathrm{O}$ radiocolóide isoladamente identificou linfonodos em 74 de 75 pacientes estudadas $(98,5 \%)$, de acordo com Sato e $\operatorname{cols}^{14}$, sendo o primeiro trabalho a demonstrar o sucesso do uso de tal substância na identificação do linfonodo sentinela. Já o corante azul patente identificou 90\% dos casos, de acordo com Simmons e cols ${ }^{15}$. A associação do radiocolóide com o corante azul patente parece ser o método de escolha (99\% contra 92\% de acurácia) se comparado ao uso do corante Azul Patente isoladamente ${ }^{13}$.

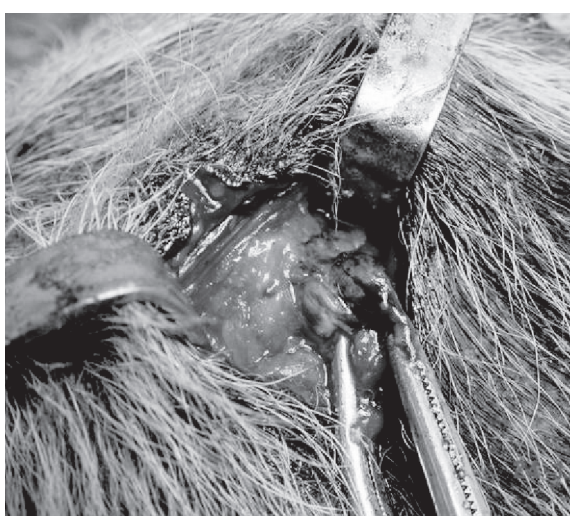

FIGURA 2 - Identificação do linfonodo sentinela da mama da cadela, corado pelo azul patente "in vivo".

\section{Conclusão}

O presente trabalho mostrou a eficiência da utilização do corante azul patente e tecnécio $99 \mathrm{~m}$ ligado à fitato, isolados ou associados, na identificação do linfonodo sentinela da região subareolar da mama dos animais estudados.

\section{Referências}

1. Moulin D. The short history of breast cancer. Kluwer: Academic Publishers; 1983.

2. Haagensen CD. The lymphatics in cancer Philadelphia: W.B. Saunders Company; 1972.

3. Cabanas RM. An approach for the treatment of penile carcinoma. Cancer 1997, 39:456-6.

4. Giuliano AE, Jones RC, Brennan M, Statman R. Sentinel lymphadenectomy in breas cancer. J Clin Oncol 1997, 15:2345-50.

5. Veronesi U, Adamus J, Bandiera, DC Brennhovd O, Caceres E, Cascinelli N, Claudio F, Ikonopisov RL, Javorski VV, Kirov S, Kulakowski A, Lacour J, Lejeune F, Mechl Z, Morabito A, Rodé I, Sergeev S, van Slooten E, Szczygiel K, Trapeznikov NN, Wagner RI. Delayed region lymph node dissection in stage I melanoma of the skin of the lower extremities. Cancer 1982 , 49:2420-30

6. Sisson, Grossman. Anatomia dos animais domésticos. 5ed. Rio de Janeiro: Guanagara Koogan; 1981.

7. Sutton R, Tsopelas C, Kollias J, Chatertton BE, Coventry BJ. Sentinel node biopsy and lymphocintigraphy with a technetium $99 \mathrm{~m}$ labeled blue dye in a rabbit model. Surgery 2002, 131 (1):44-9.

8. Veronesi U, Paganelli G, Viale G, Galimbert V, Luini A, Zurrida S, Robertson C, Sacchin V, Veronesi P, Orvieto E, De Cicco C, Intra M, Tosi G, Scarpa D. Sentinel lymph node biopsy and axillary dissection in breast

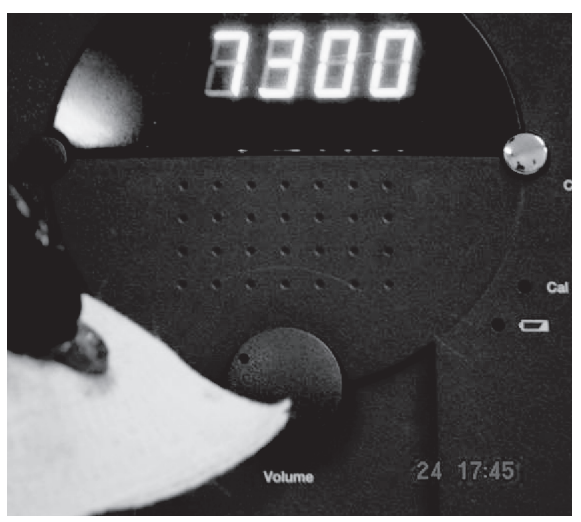

FIGURA 3 - Demonstração da atividade radioativa do linfonodo sentinela "ex-vivo", também corado por azul patente.

cancer: results in a large series. J Nat Cancer Inst 1999, 91:368-73.

9. Gershenwald JE, Thompson W, Mansfield PF, Lee JE, Colome MI, Tseng CH, Lee JJ, Balch CM, Reintgen DS, Ross MI. Multiinstitutional melanoma lymphatic mapping experience: the prognostic value of sentinel lymph node status in 612 stage I or II melanoma patients. J Clin Oncol 1999, 17:1-8.

10. Urban CA, de Lima RS, Júnior ES, Neto $\mathrm{CAH}$, Bardoe SAW. Ethics in sentinel node biopsy in breast cancer: an open question. Breast J 2002, 8(4): 253-7.

11. Tasmuth T, von Smitten K, Kalso E. Pain and other symptoms during the first year after radical and conservative surgery for breast cancer. Br J Cancer 1996, 74: 2024-31.

12. Pinheiro LGP. Linfangiossarcoma pósmastectomia (Síndrome de Stewart-Treves). Rev Col Bras Cir 1997, 24:191.

13. Cserni G, Rátjat M, Boross G, Sinkó M, Svébis M, Baltás B. Comparison of vital dye-guided lymphatic mapping and dye plus gamma probe-guided sentinel node biopsy in breast cancer. World J Surg 2002, 26(5):592-7.

14. Sato K, Uematsu M, Saito T, Ishikawa H, Tamaki K, Tamai S, Wong JR, Kusano S, Hiraide H, Mochizuki H. Sentinel node identification for patients with breast cancer using large-size radiotracer particles: technetium-99m-labeled tin colloids produced excellent results. Breast J 2001; 7(6):388-91.

15. Simmons RM, Smith SM, Osborne MP Methylene blue dye as an alternative to isosulfan blue dye for sentinel lymph node localization. Breast J 2001, 7(30):181-3.

\section{Agradecimentos}

Á Dra. Evanisa Alves Ventura diretora do Centro de Zoonoses da Prefeitura de Fortaleza por sua dedicação e reconhecimento de nosso trabalho. 
Pinheiro LGP, Moraes MO, Soares AH, Lopes AJT, Naguére MASP, Gondim FAL, Brandão CB, Nascimento DCH, Soares JPH, Silva JMM. Experimental study of the sentinel lymph node in the dog breast using blue dye and technetium Tc99m. Acta Cir Bras [serial online] 2003 Nov-Dec;18(6). Available from URL: http://www.scielo.br/acb.

ABSTRACT - The sentinel lymph node research (SLN) has been effective in the evaluation of nodal status in patients with breast cancer. A negative SLN makes an axillary lymphadenectomy unnecessary. Purpose: To identify the SLN of the subareolar region in female dog breasts using blue dye (BD), Technetium (Tc99m) or the association of both techniques and to compare their sensibility in the detection of the SLN. Methods: Seventeen female dogs were studied. 55 breasts were analyzed. DB and/or $\mathrm{Tc}^{99 \mathrm{~m}}$ were used for the identification of SLN. Tc99m was introduced two hours before the experiment. BD was introduced some minutes before the procedure. Once the SLN was localized its dissection was performed. Results: From all 44 lymph nodes in which BD was used, 40 were colored (90,90\%). Tc99m was used in 48 lymph nodes and 47 of them were radioactive $(97,91 \%$ ) (BD vs Tc99m: $\mathrm{p}=0.18 ; \mathrm{k}=$ 0.067). BD and Tc99m were associated in 37 lymph nodes, although 02 lymph nodes were not colored, all of them were radioactive (100\%)(BD vs BD+Tc99m: $\mathrm{p}=0.12 ; \mathrm{k}=0.083$; Tc99m vs BD+ Tc99m: $\mathrm{p}=1.0 ; \mathrm{k}=0.018$ ). CONCLUSION: Tc99m and BD isolated or in association, were effective in the identification of the SLN in the female dog breasts studied. KEY WORDS - Sentinel lymph node. Breast neoplasms. Surgery. Models, animal.

Conflito de interesse: nenhum

Fonte de financiamento:

Centro Regional Integrado em Oncologia

(CRIO), Fortaleza-CE

Correspondência:

Luiz Gonzaga Porto Pinheiro

Rua Vicente Leite, 2439/800

60170-151 Fortaleza - CE

Tel:(85)283-4988

luizgporto@uol.com.br

Data do recebimento: 22/08/2003

Data da revisão: $18 / 09 / 2003$

Data da aprovação: 02/10/2003

\title{
III Congresso de Urologia da-USP
}

18 a 20 de março de 2004 Centro de Convenções Rebouças, São Paulo

\author{
Secretaria Executiva: \\ Perfecta Atendimento em Eventos \\ Rua Cristiano Ribeiro da Luz Jr., 59 - São Paulo, SP \\ 05615-070 \\ Tel.: (11) 37225850 - Fax: (11) 37210970 \\ E-mail: urousp2004@ perfectaeventos.com.br \\ Site: www.perfectaeventos.com.br/eventos
}

